\title{
Verletzungen der Rotatorenmanschette, operative versus funktionell-konservative Versorgung
}

\author{
Pierre Hepp, Helmut Lill, Christoph Josten
}

\section{Zusammenfassung}

Die adäquate Versorgung von Rotatorenmanschettenverletzungen ist in erster Linie von einem differenzierten Staging abhängig. Positiv und negativ prädiktive Faktoren in der klinischen und bildgebenden Diagnostik führen schließlich zur pathologiekonformen Strategie, wobei der Übergang von der konservativ-funktionellen zur operativen Versorgung fließend ist.

\section{Einleitung}

Seit der Erstbeschreibung von Rissen im Bereich der Rotatorenmanschette vor beinahe 170 Jahren von J. G. Smith [13] sind die Erkenntnisse über Ätiologie, Pathogenese, Diagnostik und insbesondere Therapie einem ständigen Wandel unterzogen. In einer Untersuchung von Milgrom et al. [10] wurde eine expotenzielle Zunahme der partiellen bzw. kompletten Rupturen in Abhängigkeit vom ansteigendem Alter festgestellt. Es zeigte sich jedoch kein Zusammenhang zwischen dem bildmorphologischen Ausmaß der Ruptur und den Beschwerden der Patienten. Indikation, Operationszeitpunkt und Operationsverfahren werden nach wie vor kontrovers diskutiert.

\section{Anatomie - Biomechanik}

Dem M. supraspinatus, M. infraspinatus, M. subscapularis und $M$. teres minor ist

OP-JOURNAL 2002; 18: 220-224

(c) Georg Thieme Verlag Stuttgart . New York der Ursprung von der Skapula und der Ansatz als Sehnenhaube ventral, dorsal und kranial am Humeruskopf gemeinsam. Sie zeichnen sich verantwortlich für die Einzelfunktionen im Schultergelenk wie Abduktion, Adduktion sowie Außen- und Innenrotation und unterstützen die Funktion des M. deltoideus. Aus biomechanischer Sicht ist die Rotatorenmanschette als „muskulotendinöse“ Vergrößerung der Gelenkpfanne zu betrachten [15] und wird zur Lokalisation der Rotatorenmanschettenläsion von vorne nach hinten in 3 Sektoren unterteilt [7] (Abb.1).

Eine wesentliche biomechanische Funktion wird dem M. supraspinatus zugeschrieben, der mit seiner depressorischen Wirkung die Zentrierung des Humeruskopfes in die Pfanne unterstützt.

Bei der Supraspinatussehnenruptur kommt es zu einer Dezentrierung des Humeruskopfes und zu einem Anschla- gen gegen das Akromion. Zudem beträgt der Kraftanteil des M. supraspinatus bei Abduktion in der Frontalebene 35\%. Rupturen der Rotatorenmanschette führen somit zu einer Kraftschwächung unterschiedlichen Ausmaßes in den jeweiligen Bewegungsebenen.

\section{Ätiologie - Verletzungsmechanismus - Klinik}

Die Ursachen der Rotatorenmanschettenverletzung sind vielfältig und häufig nicht voneinander zu trennen. Neben vaskulärer Ursachen aufgrund einer Minderdurchblutung in der so genannten „kritischen Zone“" 0,5-1 cm proximal des Sehnenansatzes spielen degenerative Veränderungen eine wichtige Rolle. Anatomische Studien zeigten altersabhängige Verschiebungen in der Kollagenfaseranordnung sowie Alterationen in der Zellularität und Kollagenfaserstruktur, was sich in einer verminderten Reißfestigkeit mit zunehmendem Alter widerspiegelt.

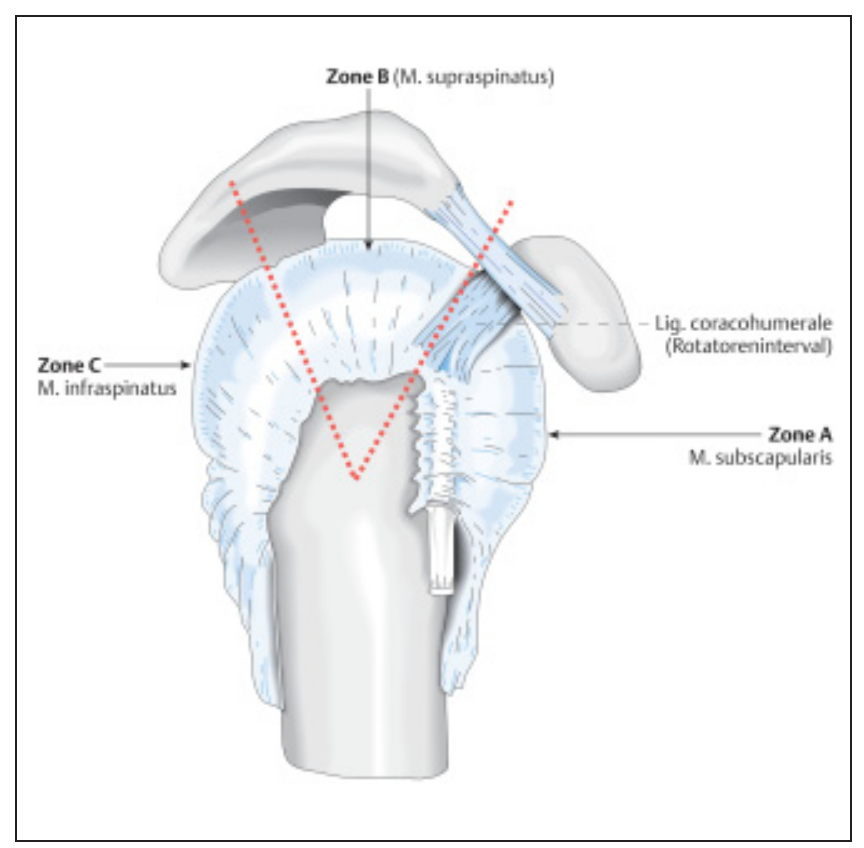

Abb.1 Die Sektoreneinteilung nach Habermeyer (aus [7]); Zone A: Subskapularis und lange Bizepssehne, Zone B: Supraspinatus, Zone C: Infraspinatus und Teres minor. 
Das typische auslösende Trauma für die akute Supraspinatussehnenruptur ist ein Sturz auf den ausgestreckten Arm oder eine erhebliche Krafteinwirkung in der entgegengesetzten Richtung der sich kontrahierenden Muskeln. Als eigene Entität erscheinen repetitive Mikrotraumen als Ursache von Rotatorenmanschettenrupturen beim Überkopfsportler. Schulterluxationen sind ebenfalls in Abhängigkeit vom Alter mit Rotatorenmanschettenläsionen vergesellschaftet. Schließlich gehören das Os acromiale und korakoidale Malformationen in den anlagebedingten Ursachenkomplex der Rotatorenmanschettenverletzung.

Das klinische Erscheinungsbild ist meistens geprägt von Schmerzen, die von der Schulter in den Arm ausstrahlen sowie einer Kraftminderung insbesondere bei Überkopfarbeiten.

Typischerweise klagen Patienten über Nachtschmerz und die Unfähigkeit, auf der betroffenen Schulter liegen zu können.

\section{Klassifikation}

Sowohl die präoperative Einteilung der Läsion als auch die perioperative Beurteilung anhand eines Klassifikationssystems sind von entscheidender Bedeutung für eine pathologiekonforme Therapieplanung, die zum Einen dem Anspruch des Patienten und zum Anderen den operativen Möglichkeiten gerecht wird.

Zunächst ist eine komplette Ruptur - also eine perforierende Kontinuitätsunterbrechung mit Verbindung zwischen Gelenkund Subakromialraum - von einer Partialruptur zu unterscheiden.

Die kompletten Rupturen werden nach Bateman eingeteilt [2]:

Grad I: klein $<1 \mathrm{~cm}$

Grad II: mittel $1-3 \mathrm{~cm}$

Grad III: groß $3-5 \mathrm{~cm}$

Grad IV: massiv $>5 \mathrm{~cm}$

Die Klassifikation nach Rowe [12] unterscheidet nach dem makroskopischen Erscheinungsbild die longitudinale (Typ 1), transversale (Typ 2), trianguläre (Typ 3) und die Massenruptur (Typ 4). Die Partialrupturen werden nach Ellman [3] in artikulärseitige (Typ A), bursaseitige (Тyp B) und intratendinöse (Typ C) Rupturen mit einer Gradierung von IIII in Abhängigkeit vom Durchmesser unterteilt. Für die arthroskopische Beurteilung hat sich die Einteilung nach Snyder
[14] durchgesetzt, wobei Kriterien verwendet werden, die sich aus der arthroskopischen Morphologie ergeben.

\section{Diagnostik - prädiktive Faktoren}

Sowohl die Anamnese und Klinik als auch die bildgebende Diagnostik können Hinweise auf die Prognose einer Rotatorenmanschettenverletzung liefern. Die klinischen Tests, wie die Impingement Tests nach Neer und Hawkins, gehören neben den aktiven und passiven Bewegungsanalysen zum Standardrepertoire der klinischen Untersuchung. Gezielte Untersuchungen, wie der „Lift-off-Test“ nach Gerber bei Subskapularisruptur oder der „Außenrotations lag-sign“ bei Infraspinatusruptur, liefern gezielte Hinweise auf die Verletzungslokalisation.

Prognostisch ungünstige Faktoren vom klinischen Standpunkt sind neben dem Alter und Nikotinabusus des Patienten der Kraftverlust bei Abduktion und bei Außenrotation des Armes sowie bei der Inspektion sichtbare Atrophie der Supraund Infraspinatussehne [6]. Deutliche Einschränkungen der passiven Gelenkbeweglichkeit haben im Gegensatz zur aktiven Beweglichkeit eine ungünstige Prognose bezüglich des Operationsergebnisses. Der präoperativ erfasste ContantScore hat in Hinblick auf die Rupturgröße und das Outcome ebenfalls eine prädiktiv prognostische Bedeutung und sollte bei der Therapieplanung miteinbezogen werden [4].

Einen besonderen Stellenwert in der Diagnostik der Rotatorenmanschettenruptur nimmt die Sonografie ein. Sie ist das einzige bildgebende Verfahren, das eine dynamische Untersuchung der Rotatorenmanschette ermöglicht und mit einer sehr hohen Genauigkeit einfache Degenerationen bis zu Komplettrupturen erfassen kann.

Zur Standarduntersuchung gehören die Nativröntgenaufnahme im a.p.-Strahlengang, sowie die axiale und die Outletview-Aufnahme. Bereits das konventionelle Röntgenbild erlaubt prognostische Aussagen über den Zustand der Rotatorenmanschette. Neben dem Vorliegen von AC-Gelenksosteophyten, Variationen im Fornix humeri und Traktionsosteophyten der Akromionunterkante hat sich der akromiohumerale Abstand (AHA) als wichtiges radiologisches Kriterium herauskristallisiert. Die Messung erfolgt in der a.p.-Aufnahme bei NeutralNull-Stellung des Armes.
Bei einem AHA größer als $7 \mathrm{~mm}$ kann von einer guten Operabilität ausgegangen werden. Bei 5-7 mm ist ein technisch schwieriger Verschluss der Rotatorenmanschette zu erwarten. Bei einem $\mathrm{AHA}<5 \mathrm{~mm}$ ist der Defekt wahrscheinlich nicht mehr verschließbar.

Einen zunehmenden Stellenwert in der Diagnostik und Prognoseabschätzung bei Rotatorenmanschettenrupturen nimmt die MRT-Untersuchung ein. Patte [11] nimmt eine Einteilung der Sehnenretraktion in drei Grade vor, die Rückschlüsse auf das funktionelle Outcome erlaubt. Von besonderem Interesse erscheint die Beurteilung der Muskelartophie bzw. Muskelverfettung in der schräg-sagittalen Bildebene nach Thomazeau [16]. In einer anatomischen und klinischen Studie schlägt er eine dreistufige Klassifikation vor, die Einfluss auf die Indikationsstellung hat. Die fettige Degeneration der Muskulatur ist irreversibel. Deshalb hängt das Heilungspotential erheblich vom Ausmaß des fettigen Umbaus ab.

Trotz aller diagnostischer Möglichkeiten ist immer das klinische Ausmaß der Beschwerden ausschlaggebend für die Therapieentscheidung. Nicht selten herrscht eine deutliche Diskrepanz zwischen Bildgebung und tatsächlichem klinischen Erscheinungsbild (Abb.2).

\section{Differenzialtherapie}

Die Indikationsstellung hängt einerseits von den positiv und negativ prädiktiven Faktoren und andererseits vom Patientenanspruch ab und ist deshalb nur nach einem ausführlichen Staging und Grading möglich [8]. Einen nicht unerheblichen Einfluss auf die Versorgungsstrategie haben persönliche Präferenzen des Operateurs und geografische Besonderheiten [17].

\section{Funktionell-konservative Versorgung}

Primäre Domäne der konservativen Behandlung sind degenerative Rotatorenmanschettenläsionen beim älteren Menschen.

Weiterhin spricht ein langsamer Beginn der Beschwerden, der inaktive Patient, eine degenerative Genese und nicht zuletzt die Non-Compliance [8] für ein konservatives Vorgehen. Im Gegensatz zur operativen Therapie bei Komplettrupturen der Rotatorenmanschette ist die konservative Vorgehensweise in der Literatur 


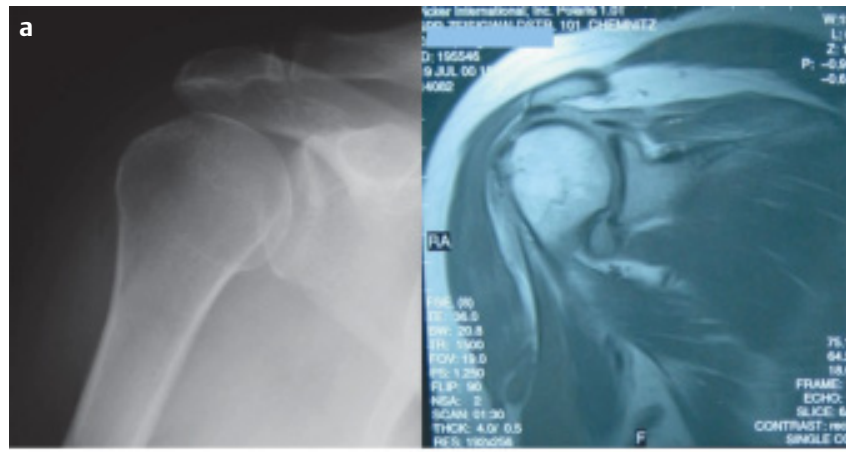

b
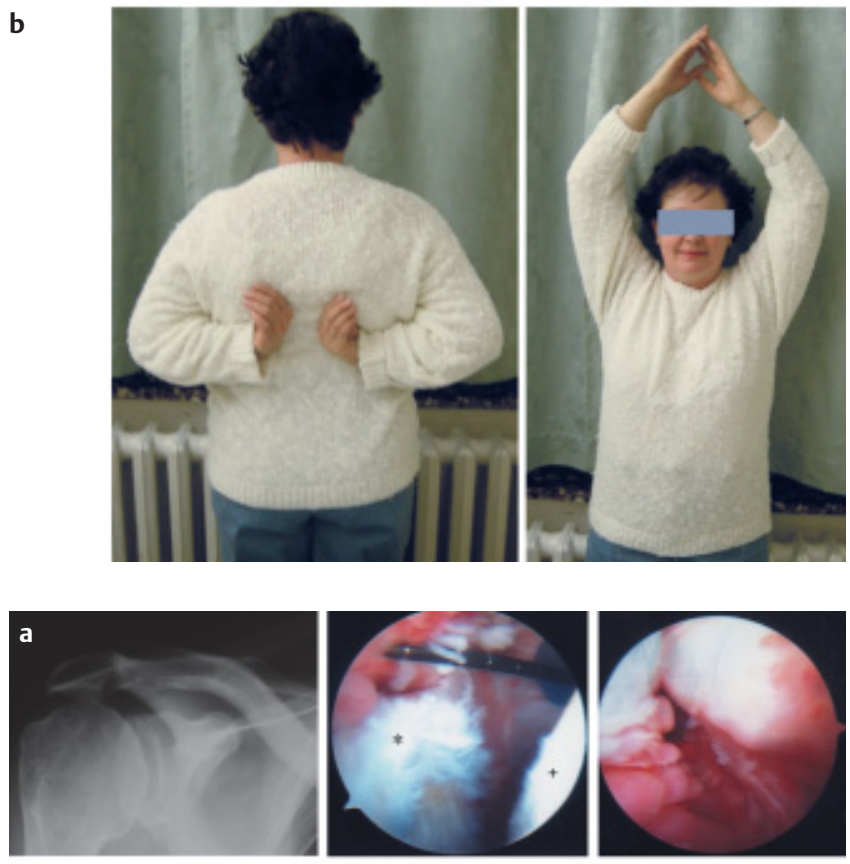

b

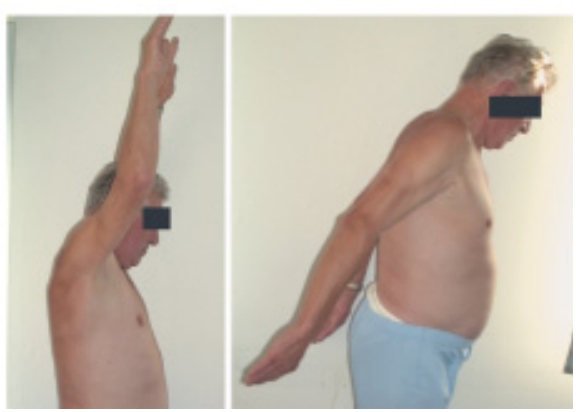

Abb. 3 76-jähriger Patient mit einer degenerativen Rotatorenmanschettenläsion. (a) links: Im a.p.-Röntgenbild AHA 5mm mit „Cuffarthropathie“. Mitte: Die arthoskopische Evaluierung zeigt eine Rotatorenmanschettenläsion Grad 4 nach Snyder mit chronischer Ruptur der SSPSehne sowie begleitende Bizepssehnenruptur $\quad{ }^{*}=$ Stumpf der langen Bizepssehne $+=$ Humeruskopf) und schwerer Synovialitis (rechtes Bild. (b): Nach erfolgtem arthroskopischen Débridement ein funktionell gutes Ergebnis (3 Monate post-op).
Abb. 2 51-jährige Patientin mit traumatischer Supraspinatussehnenruptur. (a) links: a.p.-Röntgenbild zeigt einen zentrierten Humeruskopfes und einen normalen AHA.

Rechts: Im MRT ein Retraktionsgrad Typ III nach Patte.

(b) 6 Monate nach konservativ-funktioneller Behandlung keine Schmerzen und freier Bewegungsumfang. bisher weniger betrachtet worden. Entsprechende Studien sind aufgrund unterschiedlicher Therapie-Regimes und Outcome-Analysen kaum vergleichbar [5], und Langzeitstudien zeigen, dass es sich bei der konservativen Therapie nicht um dauerhaft positive Ergebnisse handelt [9]. Grundlage jeder konservativen Behandlungsmethode muss das Verständnis über das Zusammenspiel zwischen Rotatorenmanschettenschwäche, Kapselsteifigkeit und subakromialer Enge mit begleitender Schmerzsymptomatik sein. Daraus folgend sollten folgende Grundregeln eingehalten werden:

1. Vermeiden repetetiver Bewegungsabläufe

2. Wiederherstellung der normalen Beweglichkeit über Dehnungsübungen

3. Wiederherstellung der normalen Kraft

4. Modifikation von Arbeitsabläufen

Rockwood (aus [8]) beschreibt ein dreiphasiges Konzept zur konservativen Behandlung:

1. Initiale analgetische und antiphlogistische Therapie, evtl. adjuvante Steroidinjektionstherapie mit dem Ziel, die schmerzfreie Bewegung mittels passiver Bewegungsübungen, manueller Therapie und Weichteiltechniken zu erreichen.

2. Kräftigung der Restmuskulatur, um das muskuläre Gleichgewicht zwischen Kopfdepressoren, Stabilisatoren der Skapula und M. deltoideus wiederaufzubauen.

3. Stufenweise Belastungssteigerung mit dem Ziel der Integration des Patienten in das gewohnte Arbeits- und Freizeitleben.

Als Kontraindikationen für das konservative Vorgehen gelten:

1. Massenrupturen

2. akute Rotatorenmanschettenverletzung ohne Vorschaden

3. hoher Funktionsanspruch in Sport und Freizeit

4. junge Patienten mit gesicherter Compliance

5. funktioneller Verlust der unteren Extremität oder der kontralateralen oberen Extremität

Operative Versorgung (Tab.1-4)

Das Spektrum der operativen Versorgung von Rotatorenmanschettenrupturen reicht vom einfachen Débridement (Abb.3) über Partialrekonstruktionen (Abb.4) bis hin zu aufwändigen Rekonstruktionsverfahren und Ersatzoperationen. Die Operationsindikationen ergeben 
Tab. 1 (in Anlehnung an Habermeyer und Lichtenberg [7]). SSP-Partialruptur (ohne Kontinuitätsunterbrechung)

$\begin{array}{lll}\text { gelenkseitig (Ellman A) } & \text { gelenkseitig (Ellman A) } & \text { bursalseitig (Ellman B) } \\ <50 \% & >50 \% & \text { Débridement } \\ \text { kein Akromionsporn } & \text { kein Akromionsporn } & \text { ASD } \\ \rightarrow \text { arthroskopisches } & \rightarrow \text { arthroskopische oder } & \text { (evtl. arthroskopische } \\ \text { Débridement } & \text { Mini-open-Sehnennaht } & \text { Sehnennaht oder Mini-open- } \\ & \text { mit Akromionsporn } & \text { Repair; Ellman }{\text { II \& } \text { II }^{\circ} \text { ) }} \\ & \rightarrow \text { ASD } & \end{array}$

Tab. 2 Isolierte SSP-Ruptur

Retraktion I ${ }^{\circ}$ nach Patte

Retraktion II nach Patte

Retraktion III ${ }^{\circ}$ nach Patte
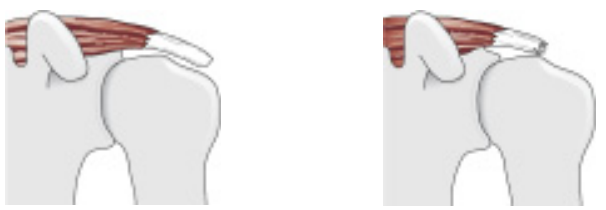

Bateman I

$\rightarrow$ arthroskopischer oder Mini-open-Repair

\section{Bateman $\mathrm{II}^{\circ}-\mathrm{III}^{\circ}$}

$\rightarrow$ arthroskopischer oder Mini-open-Repair, evtl. mit Sehnenmedialisierung

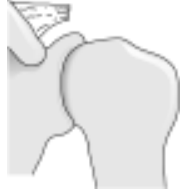

Bateman $\mathbf{I V}^{\circ}$; irreparabel

$\rightarrow$ Débridement, ASD

$\rightarrow$ Partialrekonstruktion $\rightarrow$ (Delta-Flap)

\begin{tabular}{|c|c|}
\hline $\begin{array}{l}\text { reparabel } \\
\rightarrow \text { direkte Rekonstruktion } \\
\rightarrow \text { evtl. Partialrekonstruktion } \\
\rightarrow \text { evtl. mit LBS-Tenotomie }\end{array}$ & $\begin{array}{l}\text { irreparabel } \\
\rightarrow \text { Débridement/ASD } \\
\rightarrow \text { Ersatz-Operationen } \\
\text { (Pectoralis-major-Transfer, Latissimus- } \\
\text { Transfer) }\end{array}$ \\
\hline \multicolumn{2}{|l|}{ Isolierte SSC-Ruptur } \\
\hline Reparabel & Irreparabel \\
\hline$\rightarrow$ direkte Rekonstruktion & $\rightarrow$ Pectoralis-Transfer \\
\hline
\end{tabular}

Tab. 4 Massenruptur; Verletzung $>5 \mathrm{~cm}$, Verletzung von 2 Sehnen und mehr

SSC intakt oder reparabel (AHA 5-7 mm)

$\rightarrow$ SSC-Rekonstruktion + LBS-Tenodese

+ Latissimus-dorsi-Transfer

keine Rekonstruktion möglich (AHA $<5 \mathrm{~mm}$ )

$\rightarrow$ konservativ

$\rightarrow$ Débridement/ASD, reversed ASD

$\rightarrow$ Reversed-Prothese

Abkürzungen: $\mathrm{AHA}=$ akromiohumeraler Abstand; $\mathrm{ASD}=$ arthroskopisch subakromiale Dekompression; ISP = Infraspinatus; LBS = lange Bizepssehne; SSC = Subskapularissehne; SSP = Supraspinatussehne

sich aus den Kontraindikationen der konservativen Behandlung. Zusätzlich liegt eine Reihe von Faktoren vor, die einen positiven Einfluss aus das Langzeitergebnis nach Operation aufweisen:

1. Alter $<55$

2. kein Zusammenhang zwischen Verletzungsursache und Arbeit

3. kurze Dauer der Kraftminderung

4. Nichtraucher

5. keine Steroidinjektionen
6. keine relevante Medikamentenanamnese

7. keine Infektionen

8. keine vorausgegangenen Schulteroperationen

\section{9. guter Allgemeinzustand}

Im Gegensatz zu chronischen Läsionen, wo der OP-Zeitpunkt keine Rolle spielt, werden bei frischen Rupturen die besten Ergebnisse bei Versorgung innerhalb der ersten 6 Wochen nach Trauma erreicht
[1]. Die Beschwerdedauer sollte 6 Monate nicht überschreiten, wobei jedoch die Rupturgröße den wichtigsten Einfussfaktor darstellt.

Jedem operativen Vorgehen muss eine Arthroskopie vorgeschaltet sein. Sie stellt den Gold-Standard zur Evaluierung der Rupturgröße, der Lokalisation, dem Retraktionsgrad und der Sehnenqualität dar. Die Arthroskopie ermöglicht gleichzeitig bei einer Vielzahl von Verletzungsformen die Therapie.

Habermeyer und Lichtenberg [7] haben zur Erfüllung der pathologiekonformen operativen Strategie eine Checkliste läsionsspezifischer Indikationen zur Operation entwickelt, deren zentraler Ausgangspunkt dabei der radiologisch ermittelte AHA ist.

Massenrupturen, Rerupturen und CuffArthropathien (Abb.5) zeigen den operativen Behandlungsmöglichkeiten die Grenzen auf. Ziel muss hier ein Mittelweg zwischen Schmerzreduktion und tolerabler Restbewegung sein.

\section{Literatur}

${ }^{1}$ Basset RW, Cofield RH. Acute tears of the rotator cuff. The timing of surgical repair. Clin Orthop 1983; 175: $18-24$

${ }^{2}$ Bateman JE. The diagnosis and treatment of ruptures of the rotator cuff. Surg Clin North Am 1963; 43: 1523 - 30

${ }^{3}$ Ellman H. Diagnosis and treatment of incomplete rotator cuff tears. Clin Orthop 1990; (254): 64-74

${ }^{4}$ Gazielly DF, Gleyze P, Montagnon C, Bruyere G, Prallet B. Functional and anatomical results after surgical treatment of ruptures of the rotator cuff. 2: postoperative functional and anatomical evaluation of ruptures of the rotator cuff. Rev Chir Orthop Reparatrice Appar Mot 1995; 81(1): 17-26

${ }^{5}$ Goldberg BA, Nowinski RJ, Matsen FA, 3rd. Outcome of nonoperative management of full-thickness rotator cuff tears. Clin Orthop 2001; (382): 99-107

${ }^{6}$ Habermeyer P. Die operative offene Therapie der Rotatorenmanschette. Orthopäde 1995; 24(6): $512-28$

${ }^{7}$ Habermeyer P, Lehmann L. Rotatorenmanschette, Rotatorenintervall und lange $\mathrm{Bi}$ cepssehne. In: Schulterchirurgie, Habermeyer P (Hrsg.), Urban \& Fischer, München, Jena, 2002

${ }^{8}$ Habermeyer P, Lehmann L, Lichtenberg S. Rotatorenmanschetten-Ruptur. Diagnostik und Therapie. Orthopäde 2000; 29(3): 196-208

${ }^{9}$ Itoi E, Tabata S. Conservative treatment of rotator cuff tears. Clin Orthop 1992; (275): $165-73$

${ }^{10}$ Milgrom C, Schaffler M, Gilbert S, van Holsbeeck M. Rotator-cuff changes in asymptomatic adults. The effect of age, hand dominance and gender. J Bone Joint Surg $\mathrm{Br}$ 1995; $77(2): 296-8$ 

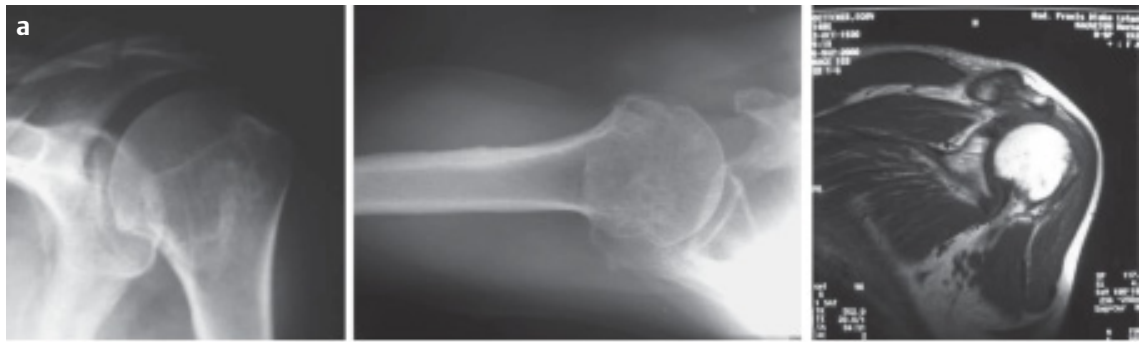

b
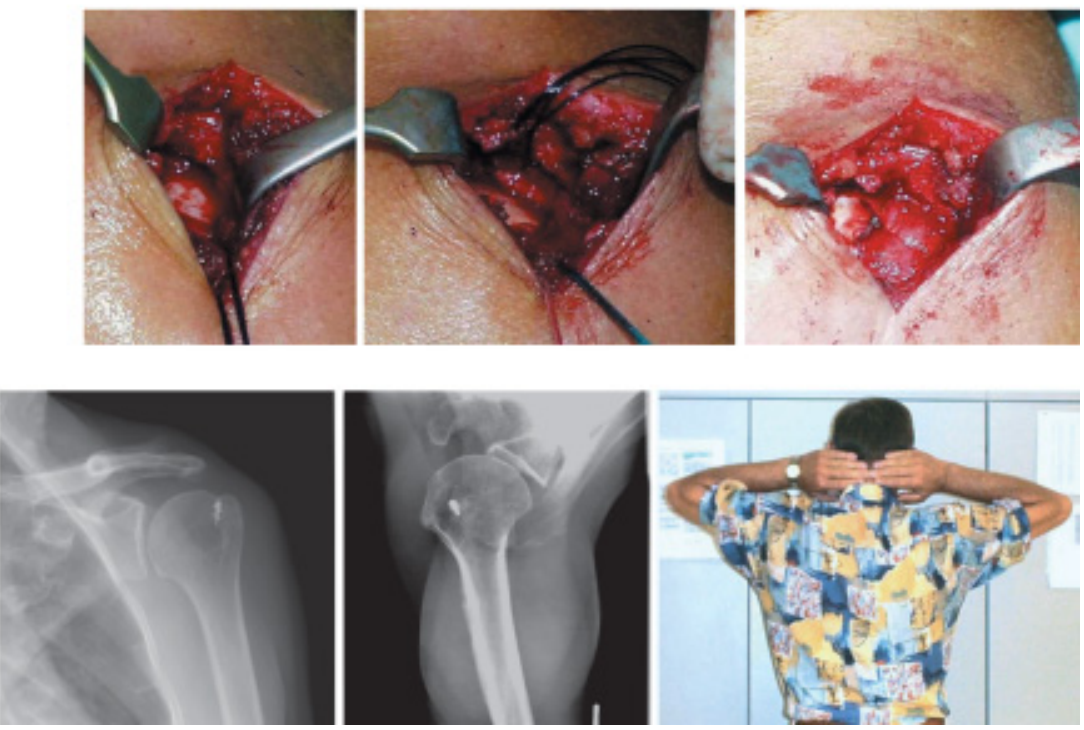

Abb. 4 69-jähriger Patient mit traumatischer Supraspinatussehnenruptur nach direktem Sturz auf die Schulter. (a) A.p. und axiale Röntgenaufnahme: AHA 6 mm, geringer Humeruskopfhochstand. Rechts: Im MRT Retraktionsgrad Typ II nach Patte. (b) Nach arthroskopischer Evaluierung (Rowe Typ II, Bateman II) Entschluss zum Mini-open-repair. Links intraoperativer Befund der Ruptur; Mitte: Platzierung des Fadenankers und Mobilisierung der SSP-Sehne; rechts: Z.n. Refixation der SSP mittels Fadenanker und modifizierter Mason-Allen-Naht (horizontale und vertikale Naht)[7]. (c) Ergebnis 10 Monate postoperativ. Links: korrekte Platzierung des Fadenankers Rezentrierung des Humeruskopfes. Rechts: gutes funktionelles Ergebnis.
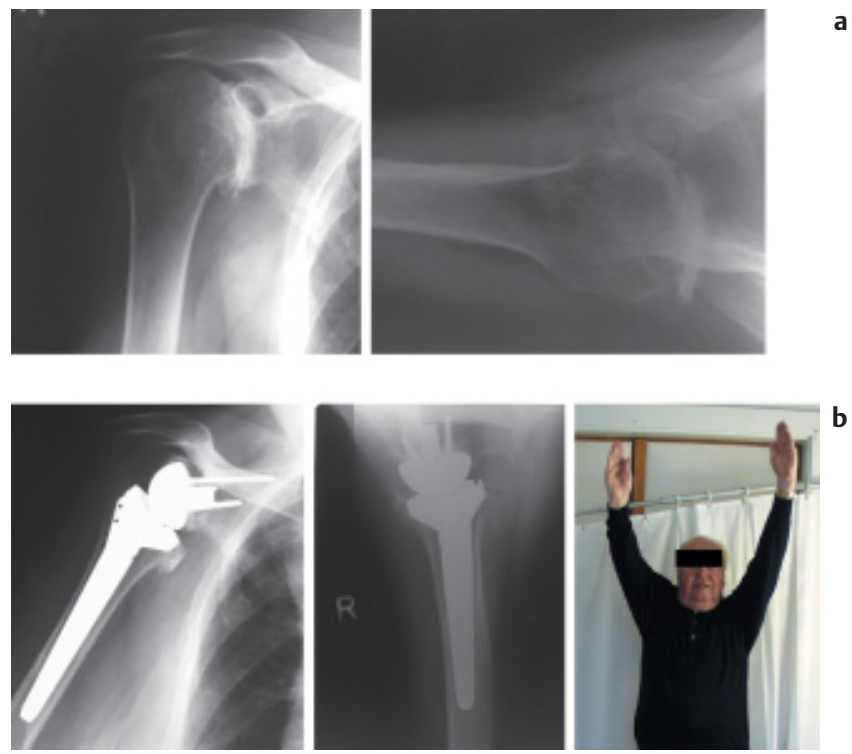

a $\quad$ Abb. 5 83-jähriger Patient mit schwerer Cuffarthropathie bei chronischer Rotatorenmanschettenmassenruptur.

(a) Glenohumerale Destruktion, Humeruskopfhochstand (AHA $<5 \mathrm{~mm}$ ).

(b) Grammont-ProFunktion 6 Monate postoperativ. these mit guter
${ }^{11}$ Patte D. Classification of rotator cuff lesions. Clin Orthop 1990; (254): 81-6

${ }^{12}$ Rowe CR. The shoulder. Churchill Livingstone, New York, Edinburgh 1988

${ }^{13}$ Smith JG. Pathological appearances of seven cases of injury of the shoulder joint, with remarks. London Med. Gazette 1834; 14: 280

${ }^{14}$ Snyder SJ, Pachelli AF, Del Pizzo W, Friedman MJ. Partial thickness rotator cuff tears: Results of arthroscopic treatment. Arthroscopy 1991; 7: $1-7$

${ }^{15}$ Südkamp NP. Die Rotatorenmanschettenruptur. Zentralbl Chir 2001; 126(3): 177-83

${ }^{16}$ Thomazeau H, Rolland Y, Lucas C, Duval JM, Langlais F. Atrophy of the supraspinatus belly. Assessment by MRI in 55 patients with rotator cuff pathology. Acta Orthop Scand 1996; 67(3): 264-8

${ }^{17}$ Vitale MG, Krant JJ, Gelijns AC, Heitjan DF Arons RR, Bigliani LU, Flatow EL. Geographic variations in the rates of operative procedures involving the shoulder, including total shoulder replacement, humeral head replacement, and rotator cuff repair. J Bone Joint Surg Am 1999; 81(6): $763-72$

\section{Dr. med. Pierre Hepp}

Assistenzarzt

Priv.-Doz. Dr. med. Helmut Lill

Oberarzt

Prof. Dr. med. Christoph Josten

Klinikdirektor

Klinik für Unfall- und Wiederherstellungschirurgie Universität Leipzig

Liebigstr. 20a

D-04103 Leipzig 